\title{
Impact of pulmonary vascular volume on mortality in IPF: is it time to reconsider the role of vasculature in disease pathogenesis and progression?
}

\author{
To the Editor:
}

The article by ЈАСОв et al. [1], reporting a computer-based computed tomography (CT) approach able to provide a precise quantification of disease extension in idiopathic publmonary fibrosis (IPF) patients by means of specific computer algorithm, CALIPER, is very interesting.

The authors show that with this algorithm it is possible to evaluate typical interstitial lung disease features, such as honeycombing, reticular pattern, ground-glass opacities and emphysema and, in addition, an innovative parameter represented by pulmonary vessel volume (PVV). CALIPER-derived measures, together with conventional visual CT scores and pulmonary function measures of disease severity, were tested for their ability to predict mortality. Surprisingly PVV, and in particular PVV5, defined as the volume of small vessels with a diameter $<5 \mathrm{~mm}^{2}$ expressed as percentage of total lung volume, at univariate analysis showed the strongest prediction ability among all considered variables. At multivariable analysis PVV, CALIPER-derived honeycombing extent and the composite physiology index were confirmed to be independent predictors of mortality and were incorporated in a new three-group staging system that was powerfully predictive of mortality in the analysed cohort of IPF patients.

The unexpected signal provided by PVV in this study was acutely discussed by the authors although it remains not fully comprehensible. The authors provide three plausible explanations related to: 1) blood-flow diversion from advanced fibrotic areas to relatively spared lung regions with aberrant dilatation of capacitance vessel resulting in increased PVV; 2) to the dilatation effect on blood vessels of increased negative pressure during inspiration due to increased lung stiffness in IPF patients; and 3) the effect of pleuro-parenchymal and bronchial-pulmonary arterial anastomosis previously described in studies performed on IPF histological lung specimens [2].

In our opinion, the results of this study could have a further explanation. Some lines of evidence suggest that vascular alterations might play a role in IPF pathogenesis and progression. In this regard, three independent histological studies [3-5] have demonstrated the presence in IPF lungs of vascular abnormalities that include aspects of pulmonary venous occlusive disease (PVOD) and aberrant capillary multiplication defined as increased alveolar septa capillary density, that are consistent with aspects of the condition known as pulmonary capillary haemangiomatosis ( $\mathrm{PCH})$. Interestingly, these alterations are consistently reported in those areas of lung parenchyma less affected by the fibrosing process and whose presence, alternately with more fibrotic and honeycombing areas, is a distinguished hallmark of the usual interstitial pneumonia pattern characterising IPF histology. Those observations may lead to speculate that vascular alterations might be the first pathological lesions in the IPF lung on which fibrosis might eventually build up by an unclear mechanism. Idiopathic forms of PVOD and PCH are uncommon causes of pulmonary hypertension, hard to distinguish from idiopathic arterial pulmonary hypertension, and are characterised by post-capillary involvement of small pulmonary venules that cause increased pressure in pulmonary capillaries, red blood cell leakage through the alveolar-capillary barrier and, as a consequence, occult alveolar haemorrhage with the accumulation of haemosiderin-laden macrophages in the alveolar spaces [6]. Differential diagnosis between other forms of pulmonary hypertension and PVOD/PCH is

@ERSpublications

Vascular disarrangement as a possible central event in disease progression in idiopathic pulmonary fibrosis (IPF) http://ow.ly/hln3307TyBk

Cite this article as: Puxeddu E, Cavalli F, Pezzuto G, et al. Impact of pulmonary vascular volume on mortality in IPF: is it time to reconsider the role of vasculature in disease pathogenesis and progression? Eur Respir J 2017; 49: 1602345 [https://doi.org/10.1183/13993003.02345-2016]. 
difficult and bronchoalveolar lavage (BAL) with haemosiderin-laden macrophage count has been proposed as an effective tool in this setting [6]. Based on this result, we recently performed a BAL study showing an increased iron burden in the alveolar milieu of IPF patients, which correlated with echocardiographic estimation of pulmonary hypertension and with disease progression and prognosis evaluated by a composite staging system [7]. Intriguingly, in a subsequent study we demonstrated an iron-dependent increased production of oxygen reactive species by alveolar macrophages of IPF patients that may represent one of the mechanisms of recurring epithelial injury thought to be involved in the fibrogenic process of the disease [8].

We believe that the discussed pieces of evidence $[3-5,7,8]$ are consistent and might represent an alternative plausible explanation of the interesting results by ЈАСОВ et al. [1], where, similarly to previous findings, the reported increased vascular volume was found in less-affected areas of the lung and showed a linear correlation with pulmonary pressure estimated by echocardiography.

The innovative results of the study by ЈАСОВ et al. [1] are of high interest not only because they offer a new powerful tool for staging IPF patients, but also because they strongly suggest that we should reconsider the current predominant "epithelial- and mesenchymal-centric" view of the disease in favour of a more inclusive view that takes into account pulmonary vascular disarrangement as a possible central event in the pathogenesis and progression of IPF.

Ermanno Puxeddu ${ }^{1,2}$, Francesco Cavalli ${ }^{1}$, Gabriella Pezzuto ${ }^{2}$, Elisa Teodori ${ }^{1}$ and Paola Rogliani ${ }^{1,2}$

${ }^{1}$ Department of Systems Medicine, University of Rome Tor Vergata, Rome, Italy. ${ }^{2}$ Respiratory Diseases Unit, Tor Vergata University Hospital, Rome, Italy.

Correspondence: Ermanno Puxeddu, Department of Systems Medicine, University of Rome Tor Vergata, Rome, Italy. E-mail: ermannopux@libero.it

Received: Nov 292016 | Accepted after revision: Dec 092016

Conflict of interest: None declared.

\section{References}

1 Jacob J, Bartholmai BJ, Rajagopalan S, et al. Mortality prediction in idiopathic pulmonary fibrosis: evaluation of computer-based CT analysis with conventional severity measures. Eur Respir J 2017; 49: 1601011.

2 Turner-Warwick M. Precapillary systemic-pulmonary anastomoses. Thorax 1963; 18: 225-237.

3 Ebina M, Shimizukawa M, Shibata N, et al. Heterogeneous increase in CD34-positive alveolar capillaries in idiopathic pulmonary fibrosis. Am J Respir Crit Care Med 2004; 169: 1203-1208.

4 Colombat M, Mal H, Groussard O, et al. Pulmonary vascular lesions in end-stage idiopathic pulmonary fibrosis: Histopathologic study on lung explant specimens and correlations with pulmonary hemodynamics. Hum Pathol 2007; 38: 60-65.

5 Kim K-H, Maldonado F, Ryu JH, et al. Iron deposition and increased alveolar septal capillary density in nonfibrotic lung tissue are associated with pulmonary hypertension in idiopathic pulmonary fibrosis. Respir Res 2010; 11: 37.

6 Rabiller A, Jaïs X, Hamid A, et al. Occult alveolar haemorrhage in pulmonary veno-occlusive disease. Eur Respir J 2006; 27: 108-113.

7 Puxeddu E, Comandini A, Cavalli F, et al. Iron laden macrophages in idiopathic pulmonary fibrosis: the telltale of occult alveolar hemorrhage? Pulm Pharmacol Ther 2014; 28: 35-40.

8 Sangiuolo F, Puxeddu E, Pezzuto G, et al. HFE gene variants and iron-induced oxygen radical generation in idiopathic pulmonary fibrosis. Eur Respir J 2015; 45: 483-490.

Copyright @ERS 2017

From the authors:

We were pleased to read the letter from E. Puxeddu and co-workers, written in response to our observation that an increased pulmonary vessel volume, derived using quantitative computed tomography (CT) analysis, strongly predicts mortality in patients with idiopathic pulmonary fibrosis (IPF) [1]. E. Puxeddu and his team highlight a selection of studies that have identified pulmonary vascular alterations in IPF, similar to those seen in pulmonary venous occlusive disease (PVOD) and pulmonary capillary haemangiomatosis $(\mathrm{PCH})[2-4]$. They suggest that when considered in the light of these previous

@ERSpublications

Understanding the complexities of the pulmonary vasculature is an important but neglected field of study in IPF http://ow.ly/anqV307U5rA

Cite this article as: Jacob J, Nicholson AG, Wells AU, et al. Impact of pulmonary vascular volume on mortality in IPF: is it time to reconsider the role of vasculature in disease pathogenesis and progression? Eur Respir J 2017; 49: 1602524 [https://doi.org/10.1183/13993003.02524-2016]. 
reports, our findings add support to the idea that vascular alterations might be the first pathological lesion in IPF preceding, and leading to, fibrogenesis.

Linkage between fibrosis and alterations in pulmonary vessel density in IPF is not a new observation. The histopathological evaluation of surgical lung biopsy samples performed by EBINA et al. [2] established that tissue surrounding regions of fibrosis in IPF is characterised by an increase in vessel profusion, whilst the fibrotic tissue itself demonstrates a reduced number of vessels. In line with these findings, fibroblastic foci, a pathological hallmark of IPF [5], have been shown to be relatively avascular [6] yet have a network of capillaries at the base of the lesion. Furthermore, within fibrotic regions of histopathological IPF samples, the normal reduction in vessel profusion with increasing distance from the alveoli spaces has been shown to be disrupted [7].

More recently, in vivo studies in animal models have highlighted the varied effects on pulmonary fibrosis mediated by modifying angiogenic pathways. In a rat model of bleomycin-induced pulmonary fibrosis, vascular endothelial growth factor (VEGF), an angiogenic mediator, was shown to reduce the severity of pulmonary hypertension yet concomitantly to increase the severity of pulmonary fibrosis [8]. In a similar mouse model, an angiostatic chemokine, CXCL11, was found to reduce total lung collagen deposition and overall fibrosis extent on morphometric analysis. However, the reduction in fibrosis, rather than being mediated through effects on pulmonary fibroblasts, was linked to reductions in numbers of pulmonary endothelial cells and the inhibition of angiogenesis [9]. Building on these reports, endostatin, a collagen degradation product that inhibits the VEGF receptor, has been shown to ameliorate bleomycin-induced pulmonary fibrosis possibly by reducing aberrant pulmonary angiogenesis and reducing alveolar epithelial cell apoptosis [10].

Despite the apparent corroboration from these various in vivo models, the translation of animal models of pulmonary fibrosis and vascular proliferation to human IPF studies must be guarded. Similarly, extrapolating relationships between the pulmonary microvasculature and fibrosis at a cellular level to the more macroscopic changes identified using quantitative CT analysis is a large, and possibly unwarranted, step. Nevertheless, dynamic or functional imaging studies may further elucidate the complex interactions that exist between the vessels in the lung and the development of fibrosis.

With regard to the specific suggestion that vascular stresses occurring secondary to either PVOD or PCH may be the first pathological lesion in the IPF lung, as E Puxeddu and colleagues state, it is worth pointing out that fibrous intimal narrowing of vessels is frequently seen on a histopathological analysis of pulmonary fibrosis, and thus adjacent vascular changes could be secondary to, or an epiphenomenon of, the fibrosing process itself. Thus it remains unclear whether vascular proliferation represents a compensatory response to subjacent pulmonary fibrosis, contributes to fibrogenesis, or represents a combination of the two.

Despite our intentional restraint in suggesting hypotheses to explain our observations about pulmonary vascular volume in IPF, the interest our work has generated is encouraging. Whilst exact relationships are elusive, it is becoming increasingly apparent that understanding the complexities of the pulmonary vasculature is an important but neglected field of study in IPF.

Joseph Jacob $^{1}$, Andrew G. Nicholson ${ }^{2}$, Athol U. Wells ${ }^{3}$ and David M. Hansell ${ }^{1}$

${ }^{1}$ Department of Radiology, Royal Brompton Hospital, Royal Brompton and Harefield NHS Foundation Trust, London, UK. ${ }^{2}$ Department of Pathology, Royal Brompton Hospital, Royal Brompton and Harefield NHS Foundation Trust, London, UK. ${ }^{3}$ Interstitial Lung Disease Unit, Royal Brompton Hospital, Royal Brompton and Harefield NHS Foundation Trust, London, UK.

Correspondence: Joseph Jacob, Department of Radiology, Royal Brompton Hospital, Royal Brompton and Harefield NHS Foundation Trust, London, UK. E-mail: akelajacob@gmail.com

Received: Dec 222016 | Accepted: Jan 032017

Conflict of interest: Disclosures can be found alongside this article at erj.ersjournals.com

\section{References}

1 Jacob J, Bartholmai BJ, Rajagopalan S, et al. Mortality prediction in idiopathic pulmonary fibrosis: evaluation of computer-based CT analysis with conventional severity measures. Eur Respir J 2017; 49: 1601011.

2 Ebina M, Shimizukawa M, Shibata N, et al. Heterogeneous increase in CD34-positive alveolar capillaries in idiopathic pulmonary fibrosis. Am J Respir Crit Care Med 2004; 169: 1203-1208.

3 Colombat $\mathrm{M}, \mathrm{Mal} \mathrm{H}$, Groussard $\mathrm{O}$, et al. Pulmonary vascular lesions in end-stage idiopathic pulmonary fibrosis: histopathologic study on lung explant specimens and correlations with pulmonary hemodynamics. Hum Pathol 2007; 38: 60-65.

4 Kim K-H, Maldonado F, Ryu JH, et al. Iron deposition and increased alveolar septal capillary density in nonfibrotic lung tissue are associated with pulmonary hypertension in idiopathic pulmonary fibrosis. Respir Res 2010; 11: 37.

5 Raghu G, Collard HR, Egan JJ, et al. An official ATS/ERS/JRS/ALAT statement: idiopathic pulmonary fibrosis: evidence-based guidelines for diagnosis and management. Am J Respir Crit Care Med 2011; 183: 788-824. 
6 Lappi-Blanco E, Kaarteenaho-Wilk R, Soini Y, et al. Intraluminal fibromyxoid lesions in bronchiolitis obliterans organizing pneumonia are highly capillarized. Hum Pathol 1999; 30: 1192-1196.

7 Renzoni EA, Walsh DA, Salmon M, et al. Interstitial vascularity in fibrosing alveolitis. Am J Respir Crit Care Med 2003; 167: 438-443.

8 Farkas L, Farkas D, Ask K, et al. VEGF ameliorates pulmonary hypertension through inhibition of endothelial apoptosis in experimental lung fibrosis in rats. J Clin Invest 2009; 119: 1298-1311.

9 Burdick MD, Murray LA, Keane MP, et al. CXCL11 attenuates bleomycin-induced pulmonary fibrosis via inhibition of vascular remodeling. Am J Respir Crit Care Med 2005; 171: 261-268.

10 Wan Y-Y, Tian G-Y, Guo H-S, et al. Endostatin, an angiogenesis inhibitor, ameliorates bleomycin-induced pulmonary fibrosis in rats. Respir Res 2013; 14: 56.

Copyright @ERS 2017 